\title{
Efecto de la temperatura sobre los valores colorimétricos de la carne de bovino estimulada eléctricamente
}

\author{
The effect of temperature on colorimetric values of electrically stimulated beef
}

\author{
E Sánchez-López ${ }^{\mathrm{a}}$, C Pérez-Linares ${ }^{\mathrm{a}}{ }^{*}$ B Herrera-Slim ${ }^{\mathrm{b}}$, A Barreras-Serrano ${ }^{\mathrm{a}}$, F Figueroa-Saavedra ${ }^{\mathrm{a}}$ \\ anstituto de Investigaciones en Ciencias Veterinarias, Universidad Autónoma de Baja California, Baja California, México. \\ ${ }^{\text {b}}$ Estudiante del programa de Maestría en Ciencias Veterinarias Instituto de Investigaciones en Ciencias Veterinarias, Universidad \\ Autónoma de Baja California, Baja California, México.
}

\begin{abstract}
SUMMARY
The effect of electrically stimulating bovine carcass on the relation between temperature and the colorimetric variables $\mathrm{L}^{*}, \mathrm{a}^{*}, \mathrm{~b}^{*}, \mathrm{C}^{*}$ and $\mathrm{H}^{*}$ was determined using a sample of 260 carcasses processed in a federal inspection type slaughter plant (TIF) located in Mexicali, Mexico. Two types of linear regression models that included a dummy variable were used, which assumed a value of 1 when the carcass was stimulated and 0 when not, the first model determined the change in intercept resulting from electrical stimulation and the second model measured the change in the slope. In the first model, the differential effect of the stimulation was estimated for $\mathrm{L}^{*}, \mathrm{a}^{*}, \mathrm{~b}^{*}, \mathrm{C}^{*}$ and $\mathrm{H}^{*}$ while in the second model the change in the dependent variable as a result of a unit change of temperature was measured. It was found that the electrical stimulation of the carcasses increased the values of $a^{*}, b^{*}$, $\mathrm{C}^{*}$ and $\mathrm{H}^{*} 24$ hours after slaughter and that the stimulated carcasses showed a higher value for the same variables as a result of temperature reduction when compared with non-stimulated carcasses. No statistical relation was found in the case of $\mathrm{L}^{*}$. Considering that electrical stimulation is a qualitative variable, regression models with dummy variables may be considered useful tools to measure its effects.
\end{abstract}

Key words: electric stimulation, $\mathrm{pH}$, colour, beef.

\section{RESUMEN}

El objetivo del estudio fue medir el efecto de la aplicación de estimulación eléctrica sobre la relación entre la temperatura de enfriamiento y las variables colorimétricas: $\mathrm{L}^{*}, \mathrm{a}^{*}, \mathrm{~b}^{*}, \mathrm{C}^{*}$ y $\mathrm{H}^{*}$ en canales de bovino. Se utilizaron 262 canales procesadas en una planta de sacrificio Tipo Inspección Federal (TIF) en Mexicali, México. Dos tipos modelos de regresión fueron usados, estos incluyeron una variable dicotómica que asumía el valor 0 cuando no se aplicaba el estímulo eléctrico a la canal y 1 cuando era estimulada. El primer modelo determinó el cambio en el intercepto que resulta de la estimulación, y el segundo midió la variación de la pendiente que es consecuencia del estímulo, de tal forma que fue posible estimar el efecto diferencial de la estimulación individualmente para $\mathrm{L}^{*}, \mathrm{a}^{*}, \mathrm{~b}^{*}, \mathrm{C}^{*}$ y $\mathrm{H}^{*}$. Además se midió el efecto marginal, que muestra cómo cambia el valor de las variables dependientes como consecuencia de un cambio unitario en la variable independiente. Los resultados sugieren que la estimulación eléctrica incrementa los valores de $\mathrm{a}^{*}, \mathrm{~b}^{*}, \mathrm{C}^{*}, \mathrm{y} \mathrm{H}^{*}$ medidos 24 horas postsacrificio y que la disminución de la temperatura en canales estimuladas eléctricamente provoca su incremento, pues la medición del efecto marginal mostró una relación inversa entre temperatura y las variables dependientes mayor que cuando no se estimula, mientras que en el caso de L* no se encontró una relación estadística. Considerando que la variable estimulación eléctrica es cualitativa, se recomienda el uso de modelos con variables dicotómicas para su evaluación.

Palabras clave: estimulación eléctrica, $\mathrm{pH}$, color, carne.

\section{INTRODUCCIÓN}

El enfriamiento de las canales tiene como principales justificaciones la inocuidad del alimento y alargar la vida de anaquel, dejando a un lado los efectos que impactan sobre la satisfacción de los consumidores como la blandura y color de la carne (Savell y col 2005), características que se ven afectadas negativamente como consecuencia por el acortamiento de la carne que resulta del enfriamiento (Moreno 2006). En lo que respecta al color, este es una característica fundamental para determinar que la carne sea aceptada por los consumidores (Nam y Ahn 2003) y en

Aceptado: 22.01.2015.

* cristinapl@yahoo.com cuanto al proceso de enfriamiento la recomendación es que para lograr el óptimo, la temperatura de la carne debe ser disminuida lo más rápidamente posible, desafortunadamente esto se contrapone a las recomendaciones de enfriamiento requeridas para alcanzar el óptimo de suavidad (Young y col 1999).

El problema del acortamiento ha sido disminuido mediante el uso de la estimulación eléctrica (EE) de las canales, ya que provoca la contracción violenta de los músculos, acelerando la glicólisis anaerobia que a su vez provoca el aceleramiento de la disminución del $\mathrm{pH}$ reduciendo el tiempo de presentación del rigor mortis (Savell y col 2005), adicionalmente se ha encontrado que la excitación eléctrica tiene un efecto positivo sobre el color y su estabilidad (King y col 2004, Simmons y col 2008). 
Para evaluar el color de la carne se utilizan principalmente los valores de L*, a* y b*( Mancini y Hunt 2005), estos fueron desarrollados para medir la refracción espectral, de tal manera que se correlacione con la evaluación visual e indican respectivamente luminosidad, retención del rojo y de amarillo (Liu y col 1996), a partir de los dos últimos valores se obtienen adicionalmente el Croma $\left(\mathrm{C}^{*}\right)$, la que es una medida de saturación que indica la pureza del color, y el ángulo de Hue $\left(\mathrm{h}^{*}\right)$, que mide la proporción de rojo o amarillo (Warris 2000).

La EE se realiza mediante la aplicación de una corriente eléctrica mediante electrodos y puede utilizarse alto o bajo voltaje y su efecto sobre el pH está más asociado a la duración del estímulo que al tipo de estimulación (Troy y Kerry 2010), además se ha reportado que los resultados son mejores cuando el estímulo se aplica dentro de los primeros 40 minutos después del sacrificio (Bendall 1984). Sin embargo, en la práctica existe una gran variabilidad de resultados, pues es afectada por variables como el proceso de enfriamiento y voltaje de estimulación, como lo demuestran las diferencias entre los resultados de los reportes, ya que por una parte se ha encontrado que la estimulación con bajo voltaje y el enfriamiento rápido mejoraba las condiciones de calidad ( $\mathrm{Li}$ y col 2010), mientras que otra investigación acerca de tipos de estimulación no encontró diferencias de calidad entre el uso de estimulación con bajo y alto voltaje (Eikelenboom y col 1985). La estimulación eléctrica de las canales de bovino influye en el color debido a la relación que presenta con el pH muscular (Wulf y Wise 1999), las canales estimuladas presentan valores de color $\left(\mathrm{L}^{*}, \mathrm{a}^{*}, \mathrm{~b}^{*}\right)$ más altos y por lo tanto la carne es más roja y brillante que la de canales no estimuladas (De la Vega 2002); además el estímulo eléctrico acelera el enrojecimiento de la carne debido al más rápido agotamiento de los sustratos y los intermediarios en los procesos oxidativos de los músculos, lo que a su vez reduce la utilización del oxígeno del aire y permite una penetración superior del mismo en la carne, aumentando así la profundidad de la capa roja de oximioglobina (Bendall 1984).

No obstante, el estímulo eléctrico no siempre resulta en un beneficio, pues si este es excesivo se pueden presentar el problema de carne pálida, suave y exudativa (PSE), como consecuencia de una muy rápida reducción del $\mathrm{pH}$ al principio del proceso postmortem en canales que presentan altas temperaturas (Troy y Kerry 2010)

La EE de las canales y la interacción entre temperatura y $\mathrm{pH}$ pueden influir significativamente en el color mediante sus efectos sobre el rango de consumo de oxígeno (Simmons y col 2008), se ha reportado que las canales de bovino estimuladas presentan valores de color $\left(\mathrm{L}^{*}, \mathrm{a}^{*}, \mathrm{~b}^{*}\right)$ más altos y por lo tanto la carne es más roja y brillante que la de canales no estimuladas (Min y col 2002, Moreno, 2006), sin embargo, otras investigaciones no coinciden con estos resultados, pues se ha reportado que en la carne de bovino el efecto puede ser bajo, ya que se encontró que la EE solo afecta L* y a* ( Li y col 2011), así como también se reporta que en trabajos realizadas en carne de ovinos (Rosenvold y Wiklund 2011) y venado (Wiklund y col 2008) la estimulación eléctrica no tiene un efecto significativo en el color.

Considerando que la aplicación de un estímulo eléctrico a una canal es catalogada como una variable cualitativa, la determinación de su efecto hace recomendable el uso de variables dicotómicas o dummies, pues estas son de gran utilidad para explicar un modelo que no es numérico (Brooks 2008), ya que este presenta variables independientes cualitativas cuyo efecto es necesario medir mediante una codificación de los dos posibles resultados con valores numéricos, esto puede lograrse dicotomizando los resultados y asignando arbitrariamente los valores de 0 y 1 a las dos diferentes posibilidades (Asteriou y Hall 2007), de forma que es posible integrar cambios discretos de una función en un modelo de regresión. Los modelos de regresión con variables dicotómicas permiten la obtención de coeficientes diferenciales que representan cuando el valor del intercepto que recibe el valor de 1 difiere del coeficiente del intercepto de la categoría base, el que recibe el valor 0 (Gujarati y Porter 2009).

\section{MATERIAL Y MÉTODOS}

El estudio se realizó en una planta de sacrificio Tipo Inspección Federal (TIF) en el municipio de Mexicali en la región norte del estado de Baja California, México, utilizando 262 canales procedentes de animales con las siguientes características: cruzas de cebú con razas europeas (cebú 30\% y europeas $70 \%$ ) con un peso promedio en pie de $400 \mathrm{~kg}$. De las canales que integraron la muestra se obtuvo información acerca de la temperatura individual 24 horas después del sacrificio, adicionalmente a partir de tres mediciones obtenidas del músculo Longissimus dorsi entre la $11^{\mathrm{a}}$ y $12^{\mathrm{a}}$ costilla se obtuvieron los valores promedio de $\mathrm{L}^{*}, \mathrm{a}^{*} \mathrm{y} \mathrm{b}^{*}$. Además se registraron los datos respecto de la estimulación o no de la canal; del total de canales estudiadas, 183 de estas fueron estimuladas eléctricamente y 79 no recibieron el estímulo. La temperatura de la canal fue obtenida mediante un termómetro digital medida en el centro del músculo Longissimus dorsi y debido a que la temperatura promedio de la canal fue de $1,26{ }^{\circ} \mathrm{C}$, el tipo de enfriamiento fue clasificado como rápido. El periodo de estudio fue dividido en dos partes abarcando el primero de ellos de marzo a abril y el segundo de mayo a junio. Los valores $a^{*}, b^{*}$ y L* se evaluaron en la superficie muscular mediante un espectrofotómetro Minolta CM-2002 (Minolta Camera Co., Ltd, Japón) utilizando un componente especular incluido (SCI), un iluminante D65 y un observador de $10^{\circ}$, el croma fue calculado como $\mathrm{C}^{*}=\left(\mathrm{a}^{* 2}+\mathrm{b}^{* 2}\right)^{1 / 2}$ y $\mathrm{H}^{*}$ fue obtenido mediante el siguiente cálculo: $\operatorname{tang}^{-1}\left(\mathrm{~b}^{*} / \mathrm{a} *\right)$ (María y col 2003).

La EE se realizó de acuerdo con el procedimiento normal utilizado por la planta de sacrificio y consiste en 
la aplicación del estímulo dentro de los tres minutos posteriores al desensibilizado mediante la colocación de los electrodos en ambos lados del cuello de la canal degollada durante 15 segundos con una intensidad de 15 a 17 voltios, utilizando un estimulador marca Jarvis modelo Es-4.

Con el fin de determinar la naturaleza y forma de la relación entre la temperatura de la canal y las variables colorimétricas, L* (lmedia) a* (amedia), b* (bmedia), $\mathrm{C}^{*}$ (cmedia) y H* (hmedia), pero asumiendo que el comportamiento de la relación es afectada por la estimulación eléctrica de la canal, se estableció un modelo de regresión con capacidad de medir el cambio del intercepto de la recta de regresión que resulta de la aplicación del estímulo eléctrico a la canal, la que al tratarse de una variable cualitativa fue incluida como una variable dicotómica o dummy, que asume el valor 0 cuando estaba ausente y 1 cuando estaba presente. Para la estimación de la diferencia en cuanto al valor del cambio en los valores colorimétricos ante una variación unitaria de temperatura entre canales no estimuladas y estimuladas (cambio marginal) se aplicó un modelo de regresión que midió la variación en la pendiente, por lo que permitió estimar este efecto marginal. La explicación matemática se presenta a continuación en el análisis estadístico.

\section{ANÁLISIS ESTADÍSTICO}

La ecuación para el modelo con la variable dicotómica de EE utilizado para determinar un cambio de intercepto fue el siguiente (Asteriou y Hall, 2007):

$$
y_{i}=\beta_{0}+\beta_{1} x_{1 i}+\beta_{2} D_{i+} u_{i}
$$

Para poder interpretar Di, se consideraron dos posibles valores de $\mathrm{D}_{\mathrm{i}}$ y como estos afectan la especificación de la ecuación. Para $D_{i}=0$ se tiene que:

$$
y_{i}=\beta_{0}+\beta_{1} x_{1 i}+\beta_{2}(0)_{i}+u_{i}=\beta_{0}+\beta_{1} x_{1 i}+u_{i}
$$

Mientras que para $D_{i}=1$ se tiene que:

$$
y_{i}=\beta_{0}+\beta_{1} x_{1 i}+\beta_{2}(1)_{i}+u_{t}=\left(\beta_{0}+\beta_{2}\right)+\beta_{1} x_{1 i}+u_{t}
$$

y por lo tanto el intercepto es diferente de $\beta_{0}$, pues es igual a $\left(\beta_{0}+\beta_{2}\right)$, por lo que con la inclusión de la variable dummy el intercepto ha cambiado, desplazando la recta de regresión hacia arriba o hacia abajo, dependiendo de la ausencia o presencia de la EE.

Para la determinación del efecto marginal (que tanto un cambio unitario en la variable independiente afecta a la dependiente) se utilizaron variables dummies que midieron el efecto en el comportamiento de la pendiente ante la presencia o no de la EE, la ecuación que representa esta situación es la siguiente (Asteriou y Hall, 2007).

$$
y_{i}=\beta_{0}+\beta_{1} X_{1 t}+\beta_{2} D_{t} X_{1 t}+u_{t}
$$

En este caso el efecto de la variable dummy puede ser dicotomizado considerando dos posibles efectos, en el caso de que $\mathrm{D}_{\mathrm{t}}=0$ se tiene que:

$$
y_{i}=\beta_{0}+\beta_{1} X_{1 t}+\beta_{2}(0) X_{1 t}+u_{t}=\beta_{0}+\beta_{1} X_{1 t}+u_{t}
$$

en el caso de que $D_{t}=1$ se tiene que:

$$
y_{i}=\beta_{0}+\beta_{1} X_{1 t}+\beta_{2}(1) X_{1 t}+u_{t}=\beta_{0}+\left(\beta_{1}+\beta_{2}\right) X_{1 I}+u_{t}
$$

Los dos modelos de variables dicotómicas seleccionados permitieron respectivamente determinar el efecto diferencial que la EE tiene sobre las variables dependientes estudiadas y comparar el cambio de los valores colorimétricos cuando varía la temperatura entre canales estimuladas y no estimuladas eléctricamente. Debido a que se trataron de modelos de regresión, una vez que son estimados la prueba para determinar si la categorización seleccionada es relevante, se basa en una distribución t (Kennedy 1998), la que en caso de que no muestre valores significativos indicará la inexistencia de una relación funcional entre las variables. Las estimaciones fueron realizadas con el programa estadístico Megastat para Excel 2007.

\section{RESULTADOS}

La estadística descriptiva de las variables obtenidas de las 262 canales y utilizadas en los modelos de regresión se muestra en el cuadro 1, y se observa que las variables presentan rangos amplios, no obstante su variación es baja. Otro hallazgo relevante es que $\mathrm{H}^{*}$ se aproxima más a $0^{\circ}$ que a $90^{\circ}$, lo que indica que la carne tiende más hacia el rojo que al amarillo, sin embargo, el bajo promedio observado para $\mathrm{C}^{*}$ indica que el producto tiende a la opacidad.

Con el fin de validar la relación entre la variable temperatura de la canal (tcanal) y las variables dependientes $\mathrm{L}^{*}, \mathrm{a}^{*}, \mathrm{~b}^{*}, \mathrm{C}^{*}$ y $\mathrm{H}^{*}$ se estimaron los modelos de regresión lineal simple correspondientes, encontrándose en todos casos, que aun cuando los valores de $\mathrm{R}$ fueron bajos $\left(0,41\right.$ para el modelo de $\mathrm{a}^{*}, 0,37$ para el modelo de $\mathrm{b}^{*}, 0,44$ para el modelo de $\mathrm{C}^{*}, 0,29$ para el modelo de $\mathrm{H}^{*}$ y 0,17 para el modelo de $\left.\mathrm{L}^{*}\right)$, los coeficientes fueron significativos $(\mathrm{P}<0,01)$.

En cuanto a los resultados de la estimación de los modelos que miden el efecto del estímulo eléctrico (ee1) sobre las variables colorimétricas se encontró que la evaluación del efecto diferencial de $\mathrm{L}^{*}$ resultó en coeficientes no significativos $(\mathrm{P}>0,10)$, por lo que se infiere que no existe diferencia en la relación de temperatura con este indicador colorimétrico entre las canales consideradas en este estudio.

Para el caso de a* los resultados de la medición del efecto diferencial de la ES son mostrados en el cuadro 2.

Como es posible observar, los coeficientes fueron significativos $(\mathrm{P}<0,01)$ independientemente de la temperatura. Las canales que no son estimuladas presentan 
un valor promedio de a* de 11,51 , mientras que las canales estimuladas presentan un valor medio de la misma variable de 12,94 $(11,51+1,43)$. La diferencia observada entre ambos casos es pequeña, pues se ubica en 1,42, pero debido a la significancia de la variable dummy se respalda la presencia del efecto positivo de la estimulación eléctrica sobre el valor de $\mathrm{a}^{*}$.

En cuanto a la estimación del efecto marginal que tiene la temperatura de canal (marg/temp) sobre el valor de $a^{*}$, considerando la aplicación o no del estímulo eléctrico, el resultado es presentado en el cuadro 3.

Basados en el valor de los coeficientes de tcanal $(\mathrm{P}<0,01)$ y marg/temp $(\mathrm{P}<0,05)$ y utilizando el valor del primero, es posible inferir que en las canales no estimuladas el incremento de un grado de temperatura disminuye el valor de $a^{*}$ en 0,7738 unidades, mientras que ese mismo incremento en las canales que reciben el estímulo provoca una baja de 0,3918 unidades que resulta de la resta del coeficiente marg/temp al de tcanal $(-0,7738+0,3820)$, el coeficiente de la variable dummy (marg/temp) indica la diferencia en la pendiente cuando las canales son estimuladas y no estimuladas, por lo que el incremento de la temperatura de la canal resulta en un efecto negativo más pequeño sobre el valor de $a^{*}$, cuando la carne es excitada eléctricamente.

En el caso de b* el cuadro 4 muestra los coeficientes estimados para determinar el efecto que la EE tiene sobre el valor promedio de este indicador del color, observándose que independientemente de la temperatura, las canales que no son estimuladas presentan un valor promedio de $\mathrm{a}^{*}$ de 6,072 unidades, mientras que las canales estimuladas presentan una media de 7,61 $(6,02+1,58)$, el diferencial es pequeño, pues es de tan solo 1,58 unidades, pero también en este caso la significancia del coeficiente para la variable dicotómica respalda un efecto elevador de $b^{*}$ de la estimulación eléctrica.

Cuadro 1. Estadísticas descriptivas para las variables $L^{*}, a^{*}, b^{*}, C^{*}, H^{*} y$ temperatura.

Descriptive statistics for variables $L^{*}, a^{*}, b^{*}, C^{*}, H^{*}$ and temperature.

\begin{tabular}{lcccccc}
\hline Estadística & $\mathrm{L}^{*}$ & $\mathrm{a}^{*}$ & $\mathrm{~b}^{*}$ & $\mathrm{C}^{*}$ & $\mathrm{H}^{*}$ & Temperatura \\
\hline Media & $39,21 \pm 0,204$ & $11,86 \pm 0,0148$ & $6,1 \pm 0,16$ & $13,55 \pm 0,193$ & $26,93 \pm 0,457$ & $1,26 \pm 0,084$ \\
Mínimo & 27,92 & 6,59 & 0,7 & 6,64 & 5,51 & $-5,6$ \\
Máximo & 48,32 & 17,70 & 13,43 & 21,47 & 44,04 & 5,6 \\
Rango & 20,39 & 11,11 & 12,73 & 14,83 & 38,52 & 11,2 \\
\hline
\end{tabular}

Cuadro 2. Estimación del efecto diferencial de la estimulación eléctrica sobre a*.

Estimation of the differential effect of electrical stimulation on $\mathrm{a}^{*}$.

\begin{tabular}{|c|c|c|c|c|c|}
\hline Variables & Coeficiente & Error estándar & $\mathrm{P}$ & I.C. inferior ${ }_{95 \%}$ & I.C. superior $95 \%$ \\
\hline intercepto & 11,5148 & 0,2876 & $<0,01$ & 10,9483 & 12,0812 \\
\hline tcanal & $-0,5116$ & 0,1010 & $<0,01$ & $-0,7104$ & $-0,3128$ \\
\hline ee 1 & 1,4259 & 0,2977 & $<0,01$ & 0,8398 & 2,0121 \\
\hline
\end{tabular}

Cuadro 3. Resultados de la estimación del efecto marginal de la temperatura de la canal sobre a*.

Estimation of the marginal effect of carcass temperature on $\mathrm{a}^{*}$.

\begin{tabular}{lccccc}
\hline Variables & Coeficiente & Error estándar & $\mathrm{P}$ & I.C. inferior 95\% $^{\text {I.C. superior }}$ 95\% \\
\hline intercepto & 12,5257 & 0,1928 & $<0,01$ & 12,1460 & 12,9055 \\
tcanal & $-0,7738$ & 0,1371 & $<0,01$ & $-1,0438$ & $-0,5038$ \\
marg/temp & 0,3820 & 0,1560 & $<0,05$ & 0,0748 & 0,6893 \\
\hline
\end{tabular}

Cuadro 4. Estimación del efecto diferencial de la estimulación eléctrica sobre b*.

Estimation of the differential effect of electrical stimulation on $b^{*}$.

\begin{tabular}{|c|c|c|c|c|c|}
\hline Variables & Coeficiente & Error estándar & $\mathrm{P}$ & I.C. inferior $95 \%$ & I.C. superior $95 \%$ \\
\hline intercepto & 6,0272 & 0,3124 & $<0,01$ & 5,4121 & 6,6423 \\
\hline tcanal & $-0,4150$ & 0,1096 & $<0,01$ & $-0,6309$ & $-0,1991$ \\
\hline ee 1 & 1,5851 & 0,3232 & $<0,01$ & 0,9486 & 2,2216 \\
\hline
\end{tabular}


Los resultados de la estimación del efecto marginal que tiene la temperatura de canal sobre el valor de $b^{*}$, considerando la aplicación o no del estímulo eléctrico, se presentan en el cuadro 5 y se observa que de acuerdo con el valor de los coeficientes de tcanal $(\mathrm{P}<0,01)$ y marg/ temp $(\mathrm{P}<0,05)$, se estima que el incremento unitario de la temperatura reduce el valor de $b^{*}$ en 0,68 unidades, y que ese mismo nivel de incremento pero en canales receptoras de la EE se traduce en una reducción del indicador hasta $0,29(-0,6865+0,3900)$.

En cuanto a $\mathrm{C}^{*}$, los resultados de la estimación del efecto del estímulo eléctrico (ee1) sobre la variable se muestran en el cuadro 6.

Al igual que para los dos indicadores previos los coeficientes fueron significativos $(\mathrm{P}<0,01)$ y se observa que independientemente de la temperatura, las canales que no son estimuladas presentan un valor promedio de $C^{*}$ de 13,14 unidades, mientras que las canales estimuladas muestran una media de $15,04(13,14+1,90)$, el valor diferencial es pequeño, pues es de tan solo 1,90 unidades, pero también en este caso la significancia del coeficiente para la variable dicotómica respalda un efecto elevador de $C^{*}$ de la estimulación eléctrica.

La estimación del efecto marginal que tiene la temperatura de canal (marg/temp) sobre el valor de $C^{*}$, considerando la aplicación o no del estímulo eléctrico, resultó en lo que se presenta en el cuadro 3.

Considerando el valor de los coeficientes de tcanal $(\mathrm{P}<0,01)$ y marg/temp $(\mathrm{P}<0,05)$, es posible inferir que en las canales no estimuladas el incremento de un grado de temperatura disminuye el valor de $C^{*}$ en 1,02 unidades, mientras que ese mismo incremento de temperatura en las canales que reciben el estímulo provoca una baja de 0,6079 unidades $(-1,0273+0,4194)$, la significancia de la variable dummy (marg/temp) indica la presencia de una diferencia de pendiente cuando las canales son estimuladas y no estimuladas.

Como es posible observar en el cuadro 8, la estimación de los coeficientes para determinar el efecto que la EE tiene sobre el valor promedio $\mathrm{H}^{*}$ resultaron significativos,

Cuadro 5. Resultados de la estimación del efecto marginal de la temperatura de la canal sobre b*.

Estimation of the marginal effect of carcass temperature on $b^{*}$.

\begin{tabular}{|c|c|c|c|c|c|}
\hline Variables & Coeficiente & Error estándar & $\mathrm{P}$ & I.C. inferior $95 \%$ & I.C. superior $95 \%$ \\
\hline intercepto & 7,1546 & 0,2101 & $<0,01$ & 5,4121 & 6,6423 \\
\hline tcanal & $-0,6865$ & 0,1494 & $<0,01$ & $-0,6309$ & $-0,1991$ \\
\hline marg/temp & 0,3900 & 0,1700 & $<0,05$ & 0,9486 & 2,2216 \\
\hline
\end{tabular}

Cuadro 6. Estimación del efecto diferencial de la estimulación eléctrica sobre $\mathrm{C}^{*}$.

Estimation of the differential effect of electrical stimulation on $\mathrm{C}^{*}$.

\begin{tabular}{lccccc}
\hline Variables & Coeficiente & Error estándar & $\mathrm{P}$ & I.C. inferior ${ }_{95 \%}$ & I.C. superior ${ }_{95 \%}$ \\
\hline intercepto & 13,1407 & 0,3684 & $<0,01$ & 12,4151 & 13,8662 \\
tcanal & $-0,7293$ & 0,1293 & $<0,01$ & $-0,9839$ & $-0,4747$ \\
ee1 & 1,9057 & 0,3813 & $<0,01$ & 1,1549 & 2,6565 \\
\hline
\end{tabular}

Cuadro 7. Resultados de la estimación del efecto marginal de la temperatura de la canal sobre $\mathrm{C}^{*}$.

Estimation of the marginal effect of carcass temperature on $\mathrm{C}^{*}$.

\begin{tabular}{lccccc}
\hline Variables & Coeficiente & Error estándar & $P$ & I.C. inferior & I.C. superior \\
\hline intercepto & I. \\
tcanal & 14,5010 & 0,2487 & $<0,01$ & 14,0114 & 14,9907 \\
marg/temp & $-1,0273$ & 0,1768 & $<0,01$ & $-1,3755$ & $-0,6791$ \\
& 0,4194 & 0,2012 & $<0,05$ & 0,0232 & 0,8156 \\
\hline
\end{tabular}

Cuadro 8. Estimación del efecto diferencial de la estimulación eléctrica sobre $\mathrm{H}^{*}$.

Estimation of the differential effect of electrical stimulation on $\mathrm{H}^{*}$.

\begin{tabular}{lccccc}
\hline Variables & Coeficiente & Error estándar & $\mathrm{P}$ & I.C. inferior ${ }_{95 \%}$ & I.C. superior ${ }_{95 \%}$ \\
\hline intercepto & 25,1615 & 0,9319 & $<0,01$ & 23,3263 & 26,9966 \\
tcanal & $-0,7094$ & 0,3271 & $<0,05$ & $-1,3535$ & $-0,0654$ \\
ee1 & 3,8175 & 0,9644 & $<0,01$ & 1,9185 & 5,7166 \\
\hline
\end{tabular}


respaldando la confiabilidad de la estimación de la diferencia de este indicador entre canales estimuladas y no estimuladas. En este caso se observó que la media de $\mathrm{H}^{*}$ para canales no estimuladas fue de 25,16 y que en las canales que sí recibieron el estímulo el valor promedio se incrementa hasta 28,9 unidades $(25,16+3,81)$.

En cuanto al efecto marginal de la temperatura para este indicador, los resultados que se presentan en el cuadro 9 permiten establecer que una más baja temperatura de la canal tendrá un mayor efecto sobre este indicador colorimétrico si la canal no es estimulada, ya que en este caso el cambio marginal equivale a una disminución de 1,47 unidades por cada incremento unitario de temperatura y como resultado del estímulo el cambio marginal negativo se ubica en tan solo $0,34(-1,4751+1,1337)$.

\section{DISCUSIÓN}

En concordancia con lo reportado en la literatura (Roeber y col 2000, Savell y col 2005), los resultados de este trabajo indican que existe una relación entre la EE y color de la canal. En el caso de la tonalidad de la carne se ha reportado que la carne de bovino estimulada eléctricamente presenta un rojo más intenso a las 24 horas (Eikelenboom y col 1985) y este mismo resultado se ha reportado para la carne de camello (Kadim y col 2009), lo que coincide con los hallazgos de esta investigación, pues se encontró evidencia de que la EE incrementó el valor de $\mathrm{H}^{*}$ como de $\mathrm{C}^{*}$ medidos a las 24 horas después del sacrificio.

La recomendación de enfriar lo más rápidamente posible las canales para disminuir el efecto negativo que el frío tiene sobre el color resulta en efectos contrarios sobre la suavidad de la carne (Young y col 1999), y además respalda evaluar la relación que existe entre esta práctica con temperatura de la canal y el color.

Debido a que la variable EE es de naturaleza cualitativa, el uso de modelos que permitan su medición hace recomendable el uso de estimaciones con mínimos cuadrados con variables dicotómicas (Gujarati y Porter 2009), y los resultados de este trabajo confirman su utilidad, ya que fue posible tanto establecer los valores diferenciales que se observan en la colorimetría de la carne ante la ausencia o presencia de la estimulación eléctrica, como definir el efecto marginal que la temperatura tiene sobre $\mathrm{a}^{*}, \mathrm{~b}^{*}, \mathrm{C}^{*}$ y $\mathrm{H}^{*}$ en canales estimuladas o no. El incremento de $\mathrm{a}^{*}$ como resultado de la estimulación eléctrica observado en el presente trabajo ha sido reportado también tanto en carne de bovino (Roeber y col 2000) como en carne de cabrito (King y col 2004) y también se ha reportado que este estímulo incrementa $b^{*}$ y $C^{*}$ y reduce $\mathrm{H}^{*}$ (Franco y col 2009). Adicionalmente se han reportado incrementos de a* y b* en el músculo Longissumus dorsi después de su estimulación temprana (Hwang y Thompson 2001), ello implica que $\mathrm{C}^{*}$ sufre el mismo efecto, esto en coincidencia con lo obtenido en esta investigación, pues se encontró que la carne de las canales estimuladas presentó una mayor intensidad del rojo y mayor brillo. Es importante hacer notar que tomando en consideración lo reportado en cuanto a valores deseables (Schmidhofer 1994), las diferencias que presentaron $\mathrm{a}^{*}$ y $\mathrm{C}^{*}$, aunque pequeñas, son consideradas como perceptibles, además es relevante considerar que los resultados obtenidos fueron 24 horas post mortem, esto no asegura que los mejores valores de color se mantengan durante periodos más largos, ya que se ha reportado que tanto la carne de bovino (Li y col 2011) como en la de borrego (Toohey y col 2008) estimuladas eléctricamente no mantuvieron la estabilidad del color inicial.

En cuanto a la relación que presenta la temperatura con las variables colorimétricas seleccionadas, el resultado de la estimación indica que esta existe y la significancia de los coeficientes y el valor del estadístico $\mathrm{F}$ respaldan la bondad de ajuste del modelo.

Lo reportado en la bibliografía respecto de que la disminución de la temperatura en canales estimuladas eléctricamente provoca un incremento de $\mathrm{C}^{*}$ y $\mathrm{H}^{*}$ (Aalhus y col 2001) fue respaldado por el presente trabajo mediante la medición del efecto marginal, el que mostró una relación inversa entre temperatura y la variable colorimétrica superior a esta misma relación cuando no se aplica el estímulo, este comportamiento fue observado para $b^{*}$ y $a^{*}$, aunque en estos casos fue más pequeño.

Tomando en cuenta los bajos valores colorimétricos medios que se observan en la carne de las canales procesadas en la planta donde se desarrolló el estudio, es posible clasificarla como corte oscuro, y considerando lo reportado en la literatura, la oscuridad y los bajos valores de $\mathrm{C}^{*}$ y a* pudieran ser explicados por el enfriamiento rápido que utiliza la planta y la temperatura alcanzada a las 24 horas, pues la literatura reporta disminuciones de $a^{*}, b^{*}$ y $C^{*}$ conforme la temperatura aumenta (Young y col 1999, Janz y col 2002), como respaldo de este comportamiento puede considerarse que en la prevención del

Cuadro 9. Resultados de la estimación del efecto marginal de la temperatura de la canal sobre $\mathrm{H}^{*}$.

Estimation of the marginal effect of carcass temperature on $\mathrm{H}^{*}$.

\begin{tabular}{lccccc}
\hline Variables & Coeficiente & Error estándar & $P$ & I.C. inferior & I5\% $_{\text {I.C. superior }}{ }_{95 \%}$ \\
\hline intercepto & 27,8568 & 0,6176 & $<0,01$ & 26,6406 & 29,0730 \\
tcanal & $-1,4751$ & 0,4392 & $<0,01$ & $-2,3400$ & $-0,6102$ \\
marg/temp & 1,1337 & 0,4997 & $<0,05$ & 0,1496 & 2,1177 \\
\hline
\end{tabular}


acortamiento por frío de las canales se ha encontrado que un método eficiente es el retraso del enfriamiento, sin embargo los posibles efectos sobre la calidad sanitaria de la carne restringe su uso (Savell y col 2005) e incrementa la utilidad de la EE como elemento de mejora de la calidad de la carne enfriada con bajas temperaturas.

Si bien existe amplio respaldo bibliográfico respecto de la relación entre temperatura y color de la carne, resulta interesante poder llevar a cabo una medición y comparación de esta relación mediante un modelo cuantitativo, pues se requieren más trabajos que midan la influencia de la EE sobre la calidad (Simmons y col 2008), en ese sentido es importante considerar que el modelo utilizado permite incluir una variable cualitativa, ya que entonces es posible no solo medir el efecto de esta variable, sino además estimar si este es suficientemente grande para tener una aplicación práctica.

Los resultados de este trabajo corroboran los reportes en cuanto a los efectos benéficos de la EE sobre el color de la carne, no obstante también indican que el tamaño de estos es pequeño y por ello no modifican la justificación principal de la EE, que es mejorar la suavidad de la carne.

El número de variables cualitativas que afectan la calidad de las canales es amplia, pues se encuentran tanto en el período de pre como de postsacrificio y por ello es importante medir su efecto, la construcción de modelos de estimación con variables dicotómicas puede ser vista como un procedimiento útil para este efecto, con la ventaja de que su complejidad es baja y la información que proporciona es amplia y de aplicación práctica.

\section{REFERENCIAS}

Aalhus JL, JAM Janz, AKW Tong, SDM Jones, WM Robertson. 2001. The influence of chilling rate and fat cover on beef quality. Can J Anim Sci 81, 321-330.

Asteriou D, SG Hall. 2007. Dummy variables. In: Asteriou D, Hall SG (eds). Applied econometrics a modern approach. Palgrave Macmillan, New York, USA, Pp 183-202.

Bendall JR. 1984. El estímulo eléctrico de las canales de los animales de abasto. En: Lawrie R (ed). Avances de la Ciencia de la Carne. Acribia, Zaragoza, España, Pp 57-83.

Brooks C. 2008. Introductory Econometrics for Finance. $2^{\text {nd }}$ ed. Cambridge University Press, Cambridge, Massachusetts, USA, Pp 88-117.

De la Vega J. 2002. Técnicas de manejo post mortem de canales y de carne. Informativo sobre carne y productos cárnicos 29, 218-226.

Eikelenboom G, FJM Smulders, H Ruderus. 1985. The effect of high and low voltage electrical stimulation on beef quality. Meat Sci $15,247-254$.

Franco J Bianchi G, O Feed, G Garibotto, F Ballesteros, O Bentancur, M Carrere, J Chiruchi. 2009. Efecto de la estimulación eléctrica de la canal sobre la calidad de la carne de vacunos de pastoreo. ITEA 4, 313-323.

Gujarati DN, DC Porter. 2009. Econometría. 5ª ed. McGraw-Hill, México D. F.

Hwang IH, Thompson J.M. 2001. Effects of pH early postmortem on meat quality in beef Longissimus dorsi muscle. Meat Sci 58, 135-144.
Janz JAM, JL Aalhus, MA Price. 2002. Meat quality of bison (Bison bison bison) Longissimus thoracis et lumborum following very fast chilling. Can J Anim Sci 82, 327-337.

Kadim IT, O Mahgoub, OW Al-Marzooqi, SK Khalaf. 2009. Effect of low voltage electrical stimulation and splitting the carcass on histochemical and meat quality characteristics of Longissimus thoracis muscle from the one-humped camel (Camelus dromedarius). J Camelid Sci 2, 30-40.

Kennedy PA. 1998. A guide to econometrics. $4^{\text {th }}$ ed. The MIT Press, Cambridge Massachusetts, USA, Pp 248-257.

King DA, KL Voges, DS Hale, DF Waldron, DA Taylor, JW Savell. 2004. High voltage electrical stimulation enhances muscle tenderness, increases aging response, and improves muscle color from cabrito carcasses. Meat Sci 68, 529-535.

Li CB, YJ Chen, XL Xu, M Huang, TJ Hu, GH Zhou. 2010. Effect of low voltage electrical stimulation and rapid chilling on meat quality characteristics of Chinese yellow crossbred bulls. Meat Sci 72, 9-17.

Li C, J Li, X Li, M Hviid, K Lundström. 2011. Effect of low-voltage electrical stimulation after dressing on color stability and water holding capacity of bovine longissimus muscle. Meat Sci 88, 559-565.

Liu Q, KK Scheller, SC Arp, DM Schaefer, M Frigg. 1996. Color coordinates for assessment of dietary vitamin $\mathrm{E}$ effects on beef color stability. J Anim Sci 74, 106-116.

Mancini RA, MC Hunt. 2005. Current research in meat color. Meat Sci $71,100-121$.

María GA, M Villarroel, C Sañudo, JL Olleta, G Gebresenbet. 2003. Effect of transport time and ageing on aspects of beef quality. Meat Sci 65, 1335-1340.

Min JS, IS Kim, YTYoon, M Lee. 2002. Real Effect of pH on CIE, L*, a* and $\mathrm{b}^{*}$ of Loins during $24 \mathrm{~h}$ Chilling of Beef Carcasses. Asian-Aust J Anim Sci 15, 279-282.

Moreno GB. 2006. Higiene e inspección de carnes. Vol I. Ediciones Díaz de Santos, Madrid, España. Pp 210-216.

Nam KC, DU Ahn. 2003. Effects of ascorbic acid and antioxidants on the color of irradiated ground beef. J Food Sci 68, 1686-1690.

Roeber DL, RC Cannell, KE Belk, JD Tatum, GC Smith. 2000. Effects of a unique application of electrical stimulation on tenderness, color, and quality attributes of the beef Longissimus muscle. J Anim Sci 78, 1504-1509.

Rosenvold K, E Wiklund. 2011. Retail colour display life of chilled lamb as affected by processing conditions and storage temperature. Meat Sci 88, 354-360.

Savell JW, BE Mueller, B E Baird. 2005. The chilling of carcasses. Meat Sci 70, 449-459.

Schmidhofer T. 1994. Métodos analíticos. En: Prandl O, Fischer A, Schimdhofer T, Sinel HJ (eds). Tecnología e Higiene de la Carne. Editorial Acribia, Zaragoza, España, Pp 723-803.

Simmons NJ, CC Daly, TL Cummings, SK Morgan, NV Johnson, A Lombard. 2008. Reassessing the principles of electrical stimulation. Meat Sci 80, 110-122.

Toohey ES, DL Hopkins, DF Stanley, SG Nielsen. 2008. The impact of new generation pre-dressing medium-voltage electrical stimulation on tenderness and colour stability in lamb meat. Meat Sci 79, 683-691.

Troy DJ, JP Kerr 2010. Consumer perception and the role of science in the meat industry. Meat Sci 86, 214-226.

Warris PD. 2000. Meat Science. An introductory text. CABI Publishing, Wallingford, UK.

Wiklund E, G Finstad, L Johansson, G Aguiar, PJ Bechtel. 2008. Carcass composition and yield of Alaskan reindeer (Rangifer tarandus taran$d u s$ ) steers and effects of electrical stimulation applied during field slaughter on meat quality. Meat Sci 78, 185-193.

Wulf DM, JW Wise. 1999. Measuring muscle color on beef carcasses using the L* a* b* color space. J Anim Sci 77, 2418-2427.

Young OA, A Priolo, NJ Simmons, J West. 1999. Effects of rigor attainment on meat blooming and color display. Meat Sci 52, 47-56. 
